\title{
EFFECT OF SPIKES INTEGRATED TO AIRFOIL AT SUPERSONIC SPEED
}

\author{
Md Akhtar Khan', Karrothu Vigneshwara ${ }^{2}$, Suresh Kukutla ${ }^{3}$, Avinash Gupta ${ }^{4}$ \\ ${ }^{\text {I} A s s i s t a n t ~ P r o f e s s o r, ~ G I T A M ~ U n i v e r s i t y-H y d e r a b a d ~}$ \\ ${ }^{2}$ Student, GNITC \\ ${ }^{3}$ Asst. Professor, GNITC \\ ${ }^{4}$ Student, GITAM University, Hyderabad
}

\begin{abstract}
The objective of this is to analyse the flow field over an aerofoil section integrated with spikes at supersonic speed (Mach number greater than 1). Use of spike integrated with aerofoil changes the flow characteristics over aerofoil and hence aerodynamic lift and drag. The experiment consists of flow visualization graphs and measurement of coefficient of aerodynamic drag and lift. Here we are using different shapes of spike like sharp edge and hemi spherical edge. In this we will compare the flow over aerofoil with spike and without spike. The flow analysis is done by using Computational fluid dynamics (CFD). CFD is the study of external flow over a body or internal flow through the body. CFD is aiding aero-dynamist to better understand the flow physics and in turn to design efficient models. In short, CFD is playing a strong role as a design tool as well as a research tool.
\end{abstract}

Keywords: NACA 651-412 airfoil, spike, Ansys Fluent, Ansys ICEM CFD, Pressure Coefficient

\section{INTRODUCTION}

While travelling at supersonic/ hypersonic speeds, the formation of detached shock wave leads to higher aerodynamic heating and drag. The boundary layer separation will immediately starts at mid of aerofoil. This leads to turbulence flow on it. Due to turbulence flow the temperature gradient and velocity gradient will be high. So the after effect may be either loss in performance or erosion/ablation of the surface of aerofoil. In order to alleviate these problems, various techniques are being studied, e.g., integration of spikes etc.[1]

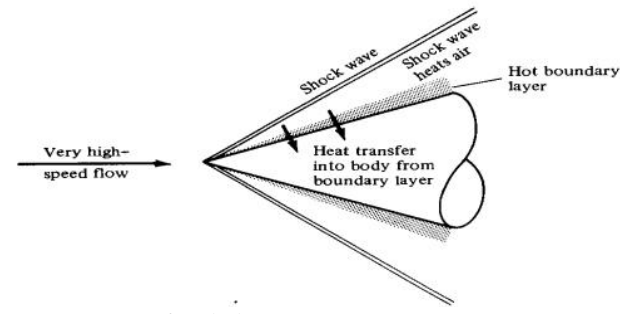

Fig 1.1 Heat Transfer [2]

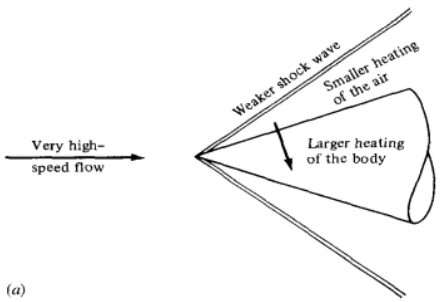

Fig 1.2 Heating of the body [2]

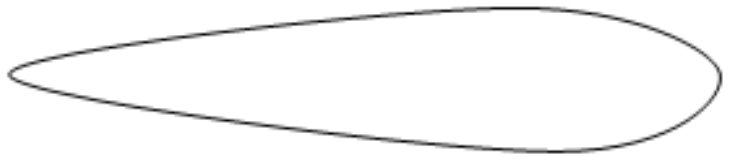

Fig 1.3 Normal

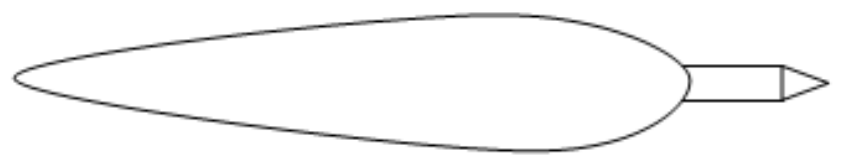

Fig 1.4 With Sharp Spike

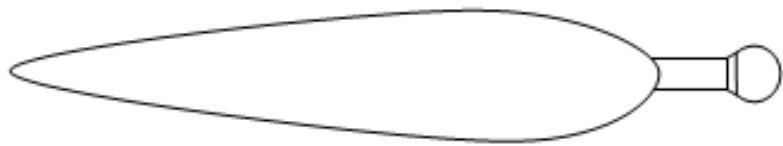

Fig 1.5 With Hemispherical Spike

Use of spike seems to be a simple preposition and it has been studied in details in last few decades. The flow field around an aerofoil will get modified due to presence of a simple spike having sharp tip. [3]

The flow field between the tip of spike and the aerofoil will strongly depend on incoming free stream flow conditions, shape of the tip of spike, length and diameter of stem which is connecting the spike tip to the aerofoil, etc. The presence of spike, leads to formation of a weaker spike shock, separated zone and separation shock due to adverse pressure gradient, recirculation zone, shear layer, etc. Due to the presence of recirculation in separated zone, major part of the aerofoil will experience lesser pressure and hence reduction in drag. 
The present investigation aims to obtain the flow field around an airfoil in the presence of either a sharp spike or hemispherical head spike at supersonic speed. Experiments and computations are made to obtain the overall flow field and drag, in the presence of either a sharp or hemispherical head spike.[4]

\subsection{Geometry}

For this study, the NACA 6-series airfoil is selected. A brief description about the NACA 6-series is given as follows. The NACA 6-Series was derived using an improved theoretical method that, like the 1-Series, relied on specifying the desired pressure distribution and employed advanced mathematics to derive the required geometrical shape. The goal of this approach was to design aerofoils that maximized the region over which the airflow remains laminar. In so doing, the drag over a small 1 range of lift coefficients can be substantially reduced. The naming convention of the 6-Series is by far the most confusing of any of the families discussed thus far, especially since many different variations exist. [6]

In this example, 6 denote the series and indicate that this family is designed for greater laminar flow than the Four- or Five-Digit Series. The second digit, 5, is the location of the minimum pressure in tenths of chord $(0.5 \mathrm{c})$. The subscript ' 1 ' indicates that low drag is maintained at lift coefficients 0.1 above and below the design lift coefficient (0.4) specified by the first digit after the dash in tenths. The final two digits specify the thickness in percentage of chord, $12 \%$. The fraction specified indicates the percentage of the aerofoil chord over which the pressure distribution on the aerofoil is uniform. If not specified, the quantity is assumed to be 1 , or the distribution is constant over the entire aerofoil.

The NACA 651-412airfoil of the NACA 6-series airfoils is selected for the present study. Two different spike shapes have been designed to integrate to the airfoil. One of the spikes has a sharp tip, while the other spike had a hemispherical tip. These spikes are attached to airfoil at the leading edge. The spike geometries are shown in the figures fig $1.6 \&$ fig 1.7

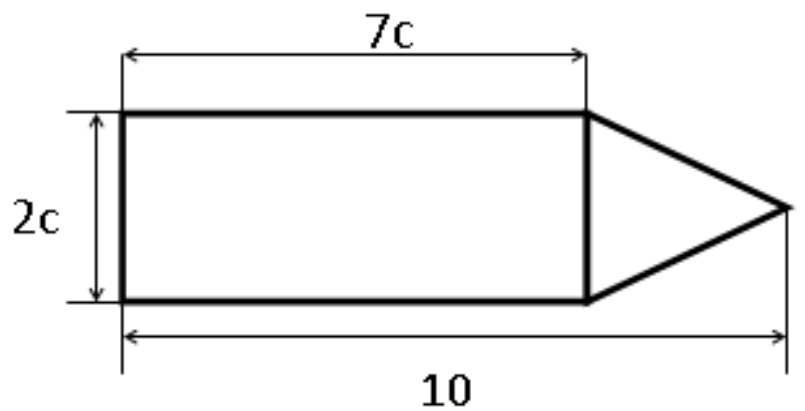

Fig 1.6 Sharp Edge

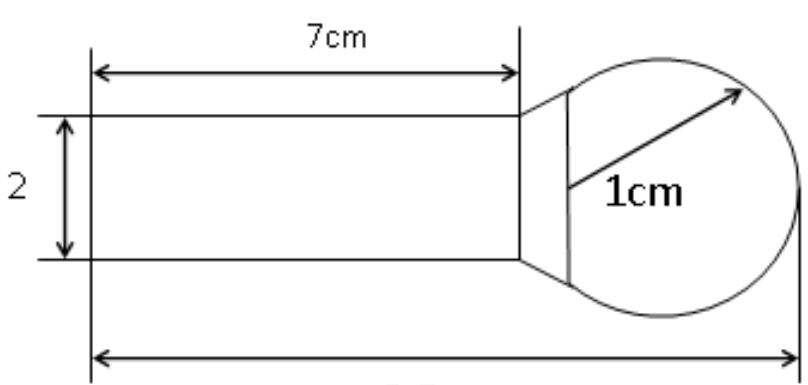

$8.5 \mathrm{~cm}$

Fig 1.7 Hemi Spherical Edge

\section{Computational Fluid Dynamics}

Computational fluid dynamics, usually abbreviated as CFD, is a branch of fluid mechanics that uses numerical methods and algorithms to solve and analyze problems that involve fluid flows. Computers are used to perform the calculations required to simulate the interaction of liquids and gases with surfaces defined by boundary conditions. With high-speed supercomputers, better solutions can be achieved. Ongoing research yields software that improves the accuracy and speed of complex simulation scenarios such as transonic or turbulent flows. Initial experimental validation of such software is performed using a wind tunnel with the final validation coming in full-scale testing, e.g. flight tests.[5]

\section{METHODOLOGY}

In all of these approaches the same basic procedure is followed.

During pre-processing

(i) The geometry (physical bounds) of the problem is defined.

(ii) The volume occupied by the fluid is divided into discrete cells (the mesh). The mesh may be uniform or non uniform.

(iii) The physical modelling is defined - for example, the equations of motions + enthalpy + radiation + species conservation

(iv)Boundary conditions are defined. This involves specifying the fluid behaviour and properties at the boundaries of the problem. For transient problems, the initial conditions are also defined.

(v) The simulation is started and the equations are solved iteratively as a steady-state or transient.

(vi)Finally a postprocessor is used for the analysis and visualization of the resulting solution.

\subsection{Software Packages Used}

\subsubsection{ANSYS ICEM CFD:}

ANSYS ICEM CFD meshing software starts with advanced $\mathrm{CAD} /$ geometry readers and repair tools to allow the user to quickly progress to a variety of geometry-tolerant meshes and produce high-quality volume or surface meshes with minimal effort. Advanced mesh diagnostics, interactive and automated mesh editing, output to a wide variety of computational fluid dynamics (CFD) and finite element analysis (FEA) solvers and multiphysics post-processing 
tools make ANSYS ICEM CFD a complete meshing solution. ANSYS endeavors to provide a variety of flexible tools that can take the model from any geometry to any solver in one modern and fully scriptable environment.[7]

(i) Mesh from CAD or faceted geometry such as STL

(ii) Efficiently mesh large, complex models

(iii) Hexa mesh (structured or unstructured) with advanced control

(iv) Extended mesh diagnostics and advanced interactive mesh editing

(v) Output to a wide variety of CFD and FEA solvers as well as neutral formats.

\subsubsection{ANSYS Fluent:}

ANSYS Fluent software contains the broad physical modelling capabilities needed to model flow, turbulence, heat transfer, and reactions for industrial applications ranging from air flow over an aircraft wing to combustion in a furnace, from bubble columns to oil platforms, from blood flow to semiconductor manufacturing, and from clean room design to wastewater treatment plants. Special models that give the software the ability to model in-cylinder combustion, aero acoustics, turbo machinery, and multiphase systems have served to broaden its reach.

ANSYS Fluent is the first commercial CFD code to provide innovative ad joint solver technology. This tool provides optimization information that is difficult or cumbersome to determine. Because it estimates the effect of a given parameter on system performance - prior to actually modifying the parameter — the ad joint solver further increases simulation speed and, more importantly, contributes to innovation. [5, 7]

The integration of ANSYS Fluent into ANSYS Workbench provides users with superior bi-directional connections to all major CAD systems, powerful geometry modification and creation with ANSYS Design Modeller technology, and advanced meshing technologies in ANSYS Meshing. The platform also allows data and results to be shared between applications using an easy drag-and-drop transfer, for example, to use a fluid flow solution in the definition of a boundary load of a subsequent structural mechanics simulation.

The combination of these benefits with the extensive range of physical modelling capabilities and the fast, accurate CFD results that ANSYS Fluent software has to offer results in one of the most comprehensive software packages for CFD modelling available in the world today.

\section{EXPERIMENTAL PROCEDURE}

\subsection{Geometry Generation}

- $\quad$ File $\rightarrow$ Import Geometry $\rightarrow$ Formatted Point Data

Select the file containing the coordinates of the airfoil and give apply as shown in below fig 3.1(a)

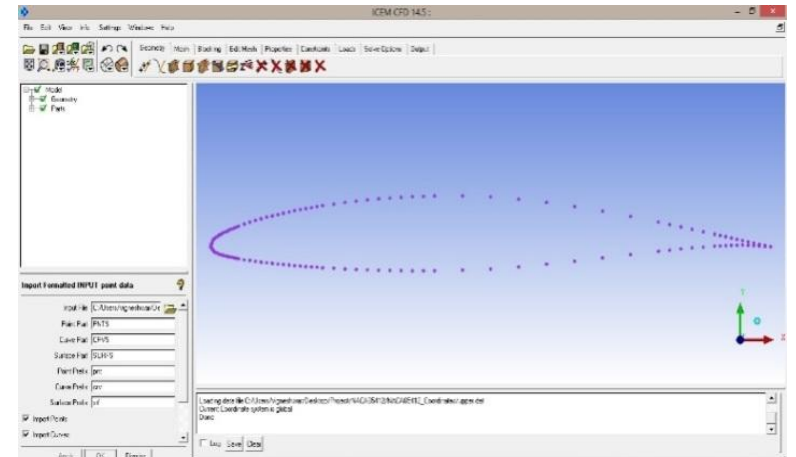

Fig 3.1(a) Imported co-ordinates of arifoil

- $\quad$ Geometry $\rightarrow$ Create/Modify Curve $\rightarrow$ From Points Select the points continuously with left click and press middle button when done. Press right click for cancel. Create Upper and Lower curves separately. Upper curve formation is shown in fig 3.1(b) and lower curve formation is shown in fig 3.1(c)

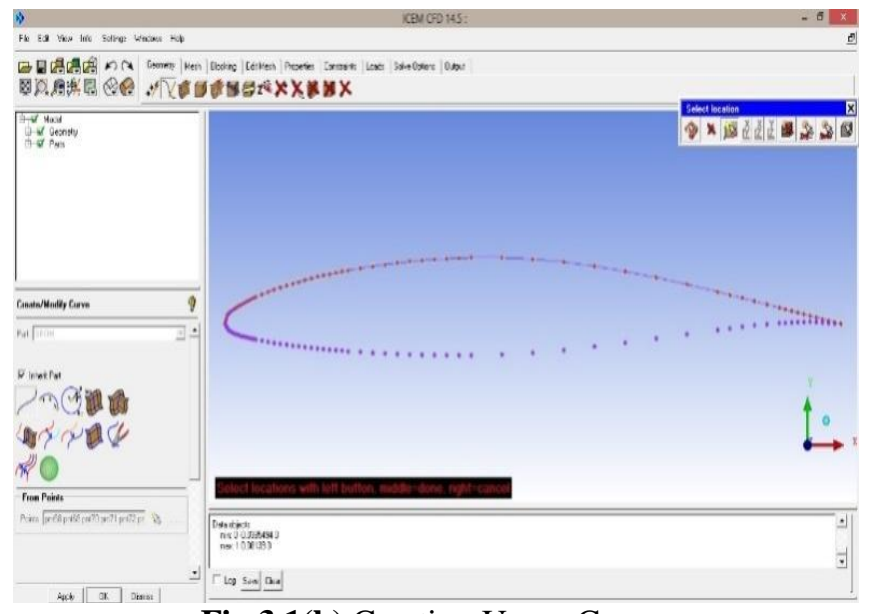

Fig 3.1(b) Creating Upper Curve

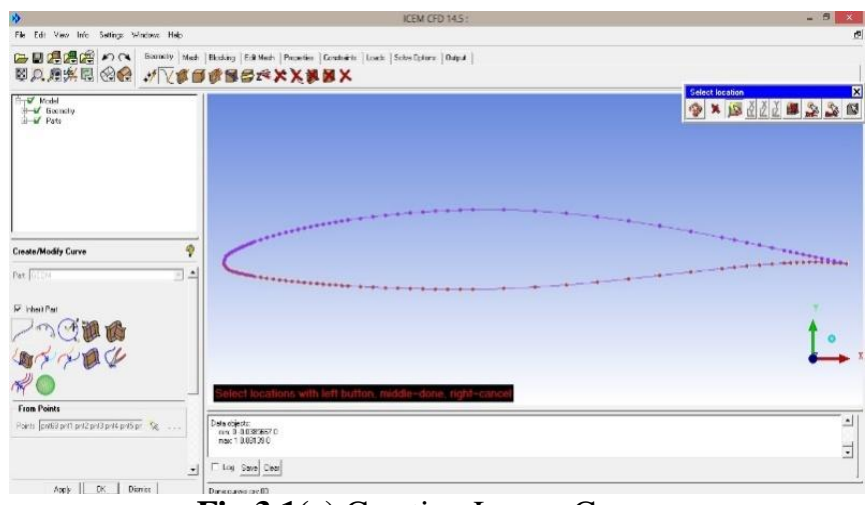

Fig 3.1(c) Creating Lower Curve

- $\quad$ Geometry $\rightarrow$ Create Points $\rightarrow$ Explicit Coordinates For creating farfiled generate the points using explicit coordinates under create points command. The points generated are shown in below Fig 3.1(d) 


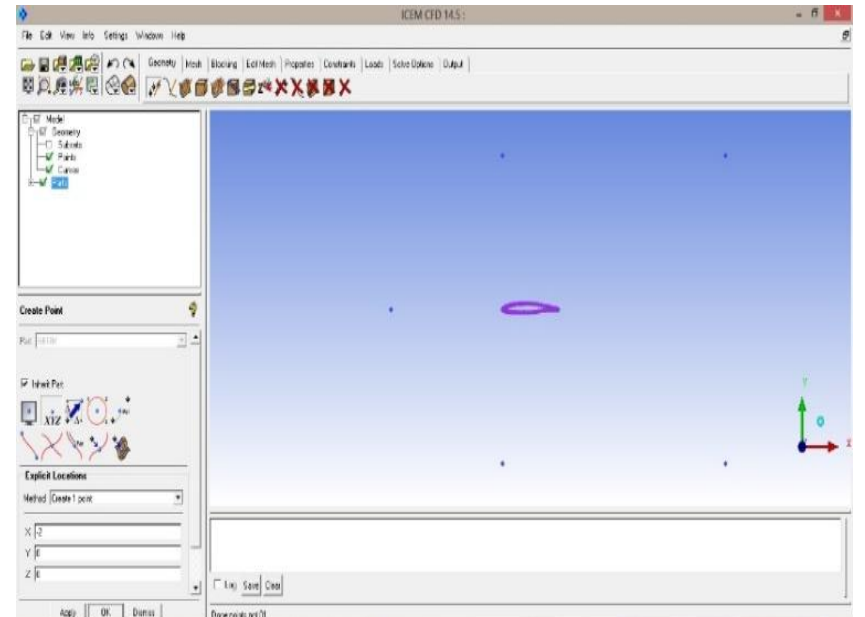

Fig 3.1(d) Creating Farfield Points

\section{- $\quad$ Geometry $\rightarrow$ Create/Modify Curve $\rightarrow$ Arc / From} Points

Create front semi-circle curve using Arc command by clicking three points continously. The other three curves are created by using From points command as shown in Fig 3.1(e).

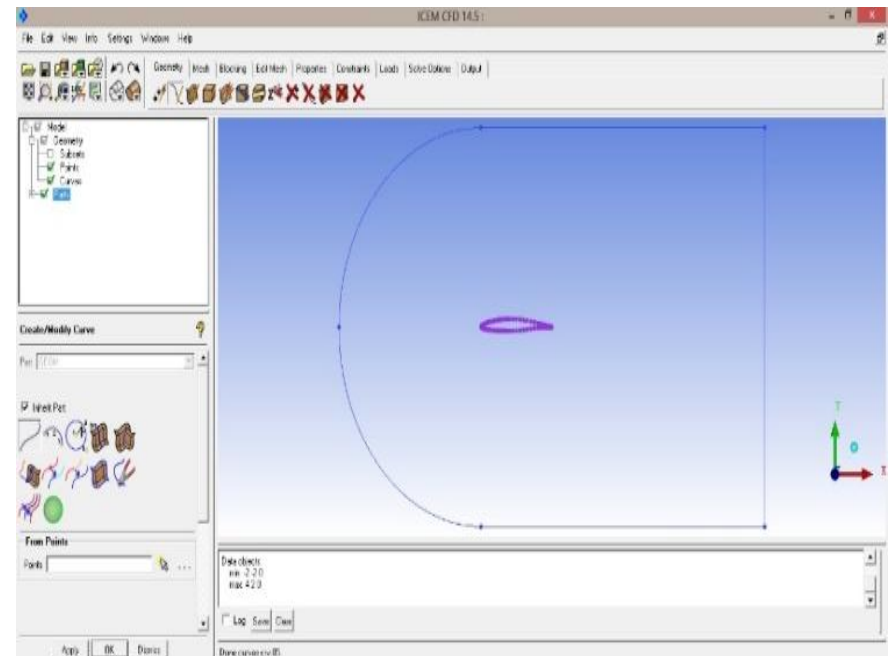

Fig 3.1(e) Creating Farfield Curves

\section{- $\quad$ Part $\rightarrow$ Right Click $\rightarrow$ Create Part}

The entities are classified into different parts like Farfield, Upper, Lower here. Right click on the Part Command in flow tree and select create part as shown in Fig 3.1(f). Give the part name and select the entities and then give apply for creating a new part as shown in fig $3.1(\mathrm{~g})$.

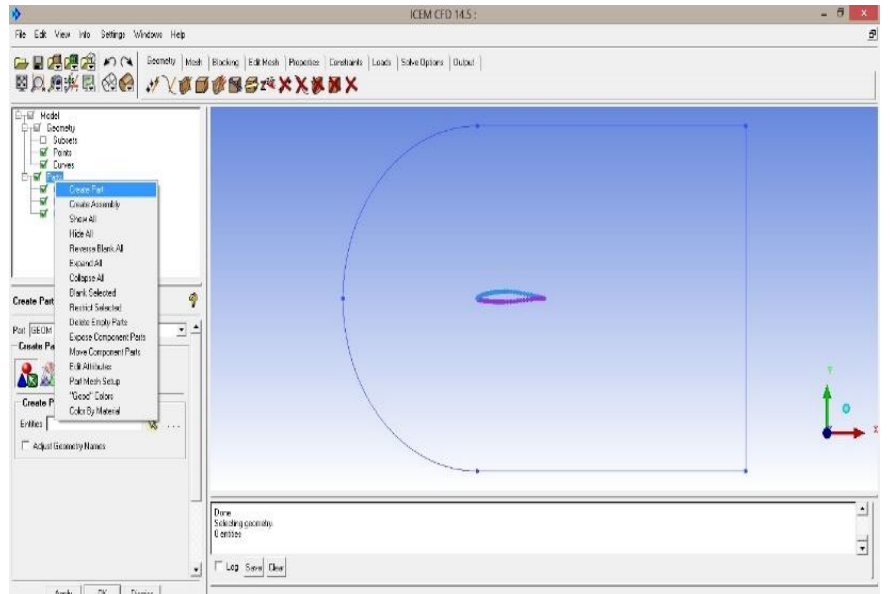

Fig 3.1(f) Classification of Different Parts

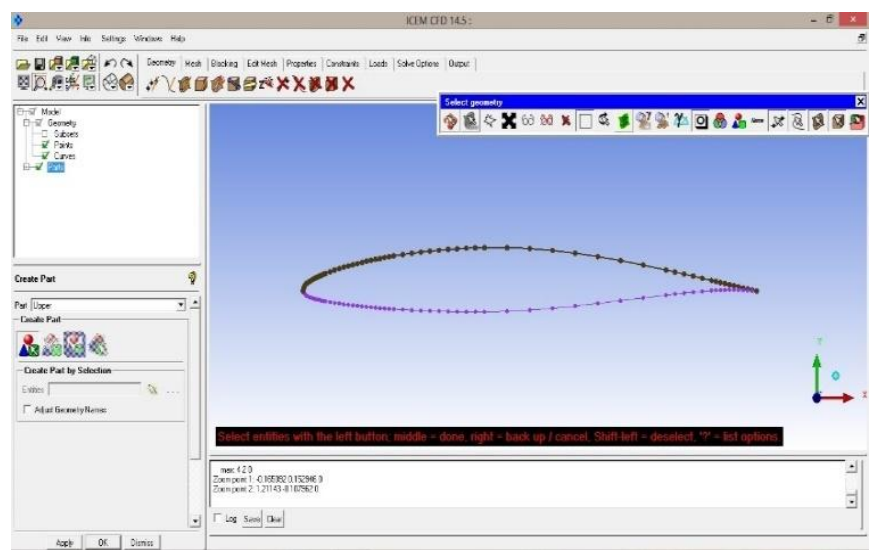

Fig 3.1(g) Creating Upper Part of Airfoil

Give name as UPPER and select the points and curve on upper side of airfoil and give apply. We can see a new part UPPER created in flow tress under Parts section. Simillarly do it for the Lower also. The remaining part can be renamed into Farfield by right click on Geom and rename command and is shown in the below fig $3.1(\mathrm{~h})$

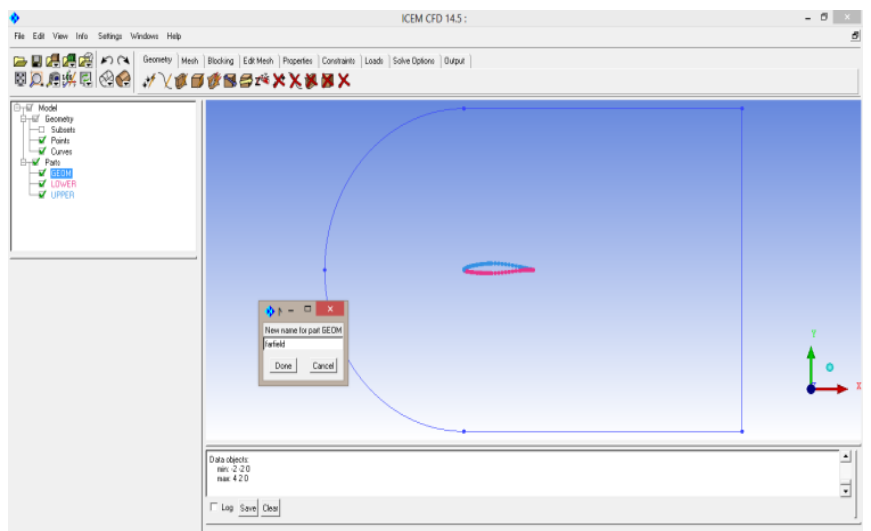

Fig 3.1(h) Renaming Geom to Farfield

- $\quad$ File $\rightarrow$ Geometry $\rightarrow$ Save Geometry as

Now save the geometry generated by fowolling the above path as shown in fig 3.1(i). The ICEM CFD saves the geometry in .tin file by default. We can directly open this tin file from here on. 


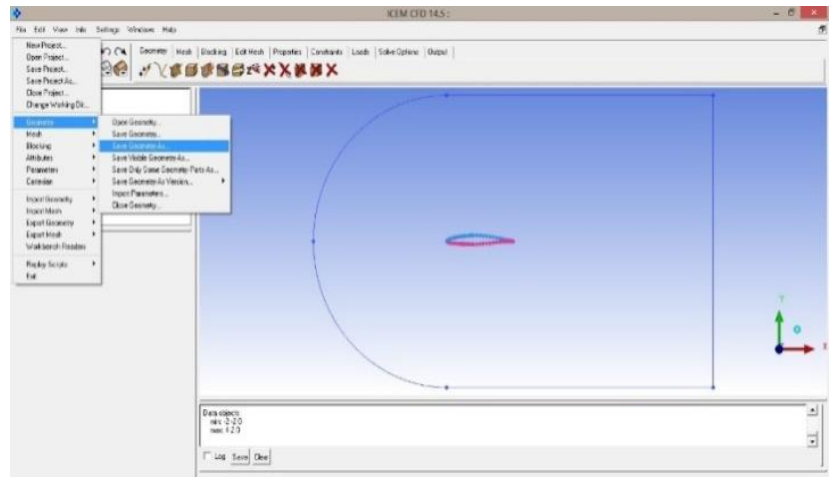

Fig 3.1(i) Saving Geometry

\subsection{Airfoil Blocking}

\section{- $\quad$ Blocking $\rightarrow$ Create Block $\rightarrow$ 2D Planar}

The Blocking is done for generating a structured mesh. In blocking the geometry shape is captured by the edges splitted to form the shape of geometry. The steps are shown above to go to blocking and is seen in below window fig 3.2(a). The rectangular initial block is formed.

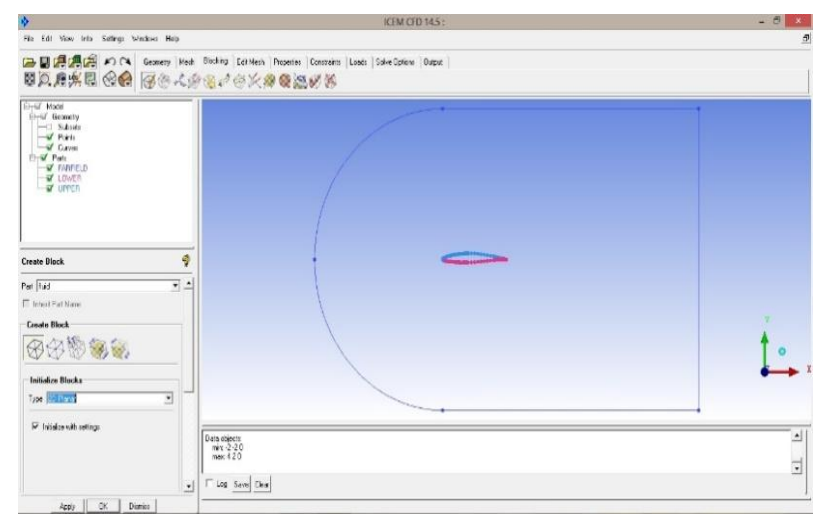

Fig 3.2(a) Blocking

- $\quad$ Blocking $\rightarrow$ Associate $\rightarrow$ Edge to Curve $\rightarrow$ Select Edge $\rightarrow$ Middle Click $\rightarrow$ Select Curve $\rightarrow$ Middle click

The initial block's 4 edges are now associated to the 4 curves of the farfield using the above command. The associated edges turn into different color. This means that the edges represent the curves. What ever conditions we impose on the edges will reflect on the curves.

\section{- $\quad$ Blocking $\rightarrow$ Associate $\rightarrow$ Snap all $\rightarrow$ All Visible $\rightarrow$ Apply}

The associated edges will take the shape of the curves automatically, if the project vertices option is highlighted during association step. If its not highlighted then we need to follow above command line to make the edges follow curve shape. The snap function will make the edges be allign with curves in best possible way as shown in fig. 3.2(b)

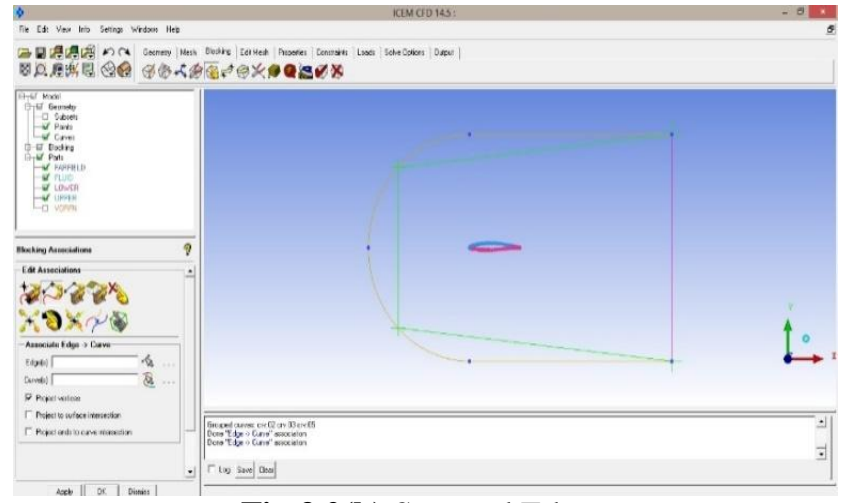

Fig 3.2(b) Snapped Edges

\section{- $\quad$ Blocking $\rightarrow$ Split Block $\rightarrow$ Create O-grid}

The o-grid is to be generated in order to capture the edges smoothly and to get good mesh quality. We can go to the ogrid menu by following the above command line. Select the block and the edge which is to be fixed. The selection will be highlighted as shown in fig 3.2(c). Give apply to see a inner block created with contant distance just inside the outer block.

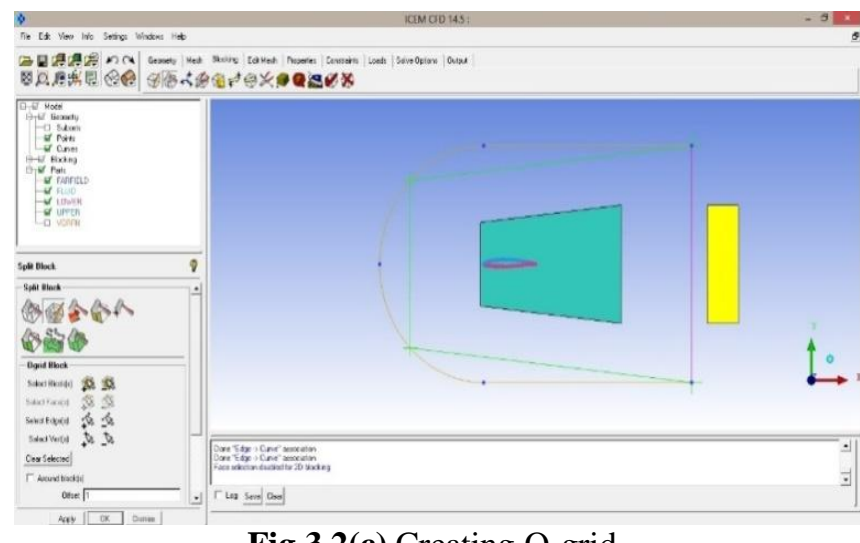

Fig 3.2(c) Creating O-grid

\section{- $\quad$ Blocking $\rightarrow$ Split Block $\rightarrow$ Split Block}

Now the block has to be split so that the airfoil can be captured. Just click at the place where we need a split. The split can be created either vertical or horizontal depending on the requirement. For now we are making a split at trailing edge of the airfoil as shown in below fig 3.2(d)

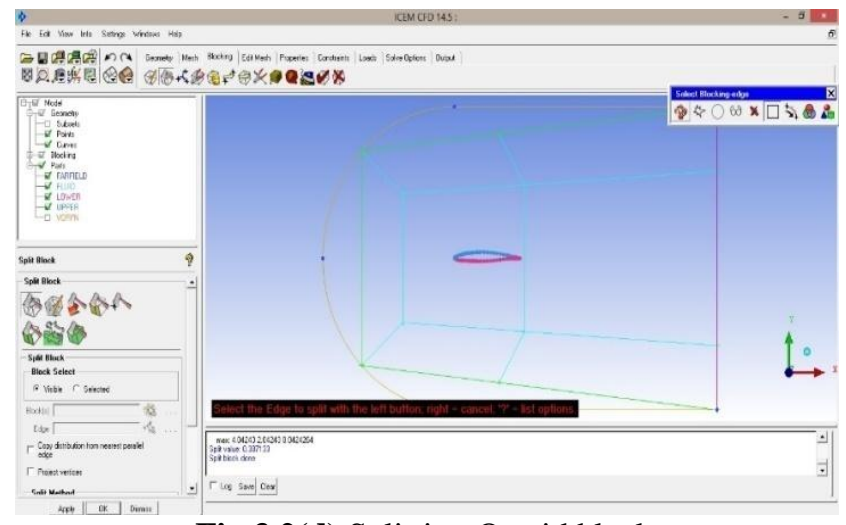

Fig 3.2(d) Splitting O-grid block 


\section{- $\quad$ Blocking $\rightarrow$ Associate $\rightarrow$ Edge to Curve}

Now associate the inner block upper edge with upper curve, inner block lower edge with lower curve and front edge to both the upper and lower curves as show in below fig 4.2(e). The Association details can be seen by right clicking on edge option on flow tree and selecting show association.

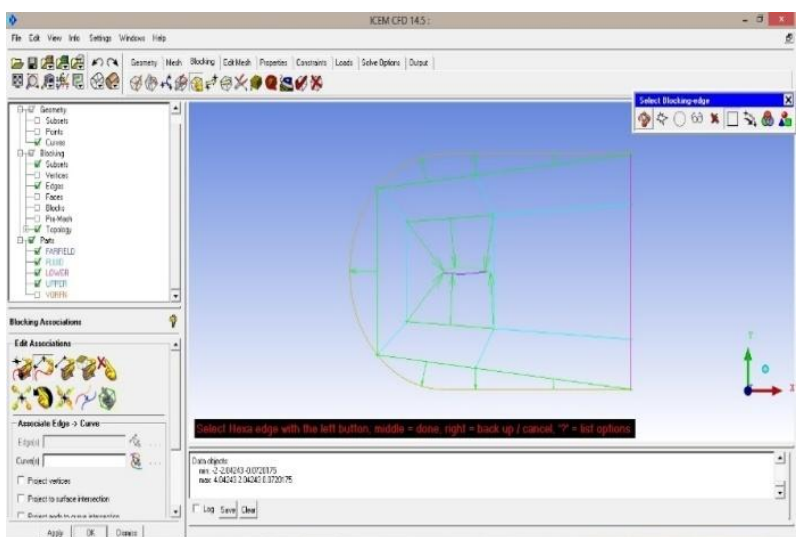

Fig 3.2(e) Showing Association of edges to curves

Now the snap all option is used to make the associated edges take the shape of upper and lower curves. The airfoil is having a sharp trailing edge. So the other unassociated edge of the inner block is not necessary. It has to be collapsed following the below command line to capture the trailing edge of the airfoil.

\section{- $\quad$ Blocking $\rightarrow$ Merge Vertices $\rightarrow$ Collapse Blocks}

For collapsing edge first select the edge to be collapsed and then the block as shown in below fig 4.2(f). Proceed and give apply so that the block will collapse along the edge length, forming a straight line as shown in below fig 4.2(g)

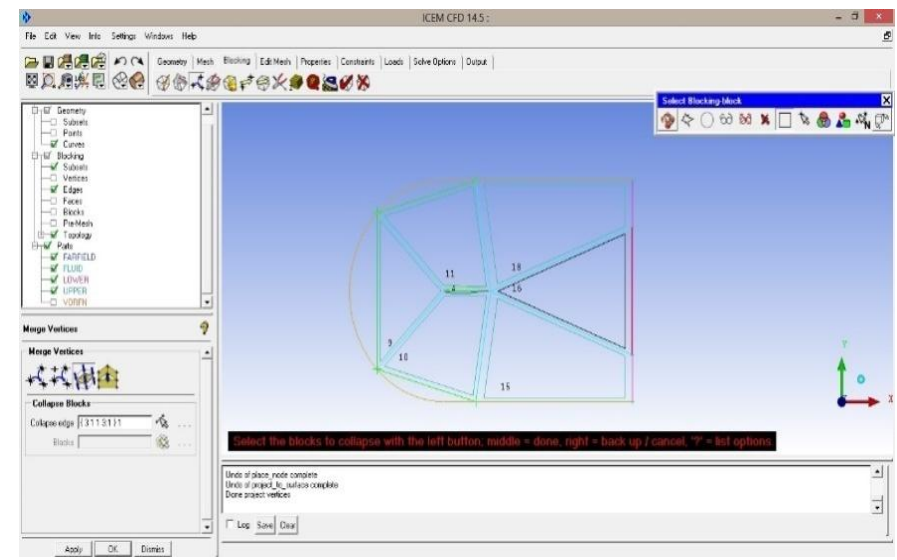

Fig 3.2(f) Collapsing rear block

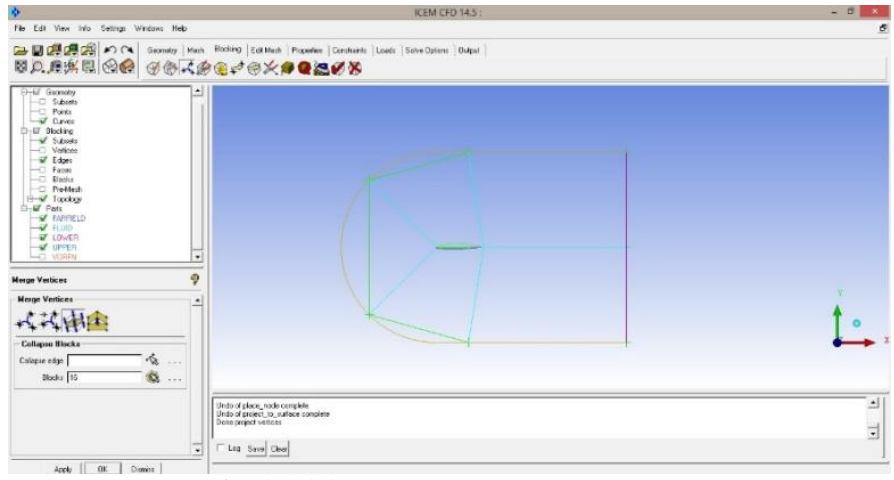

Fig 3.2(g) Collapsed block view

The block inside the airfoil should be deleted by using the following command line. This block should be deleted so that mesh will be generated, capturing the airfoil curves. The existing blocks only will get divided into nodes.

\section{- $\quad$ Blocking $\rightarrow$ Delete Block $\rightarrow$ Select Block $\rightarrow$ Apply - $\quad$ Blocking $\rightarrow$ Split Block $\rightarrow$ Split Block}

Create a split in front of the blocking as shown in the below fig 3.2(h). Create another split so that it will form a c shaped edge through out the airfoil till the end as shown in fig 3.2(i). These splits will be helpful to improve the quality of the mesh, which will be discussed later.

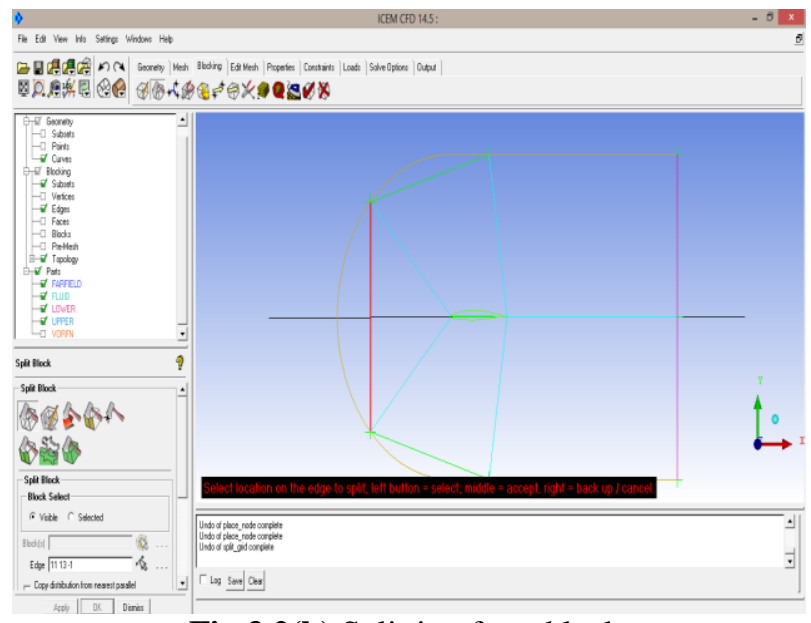

Fig 3.2(h) Splitting front block

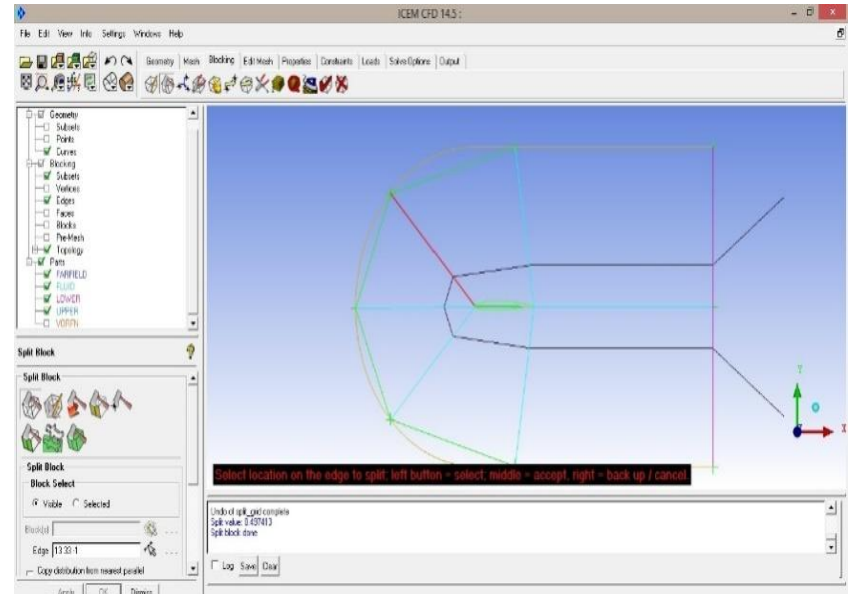

Fig 3.2(i) Splitting Edges along the Axis 


\section{- $\quad$ File $\rightarrow$ Blocking $\rightarrow$ Save Blocking as}

Now save the Blocking generated by fowolling the above path as shown in fig 4.2(j). The ICEM CFD saves the blocking in .blk file by default. We can directly import this .blk file for the geometry here on.

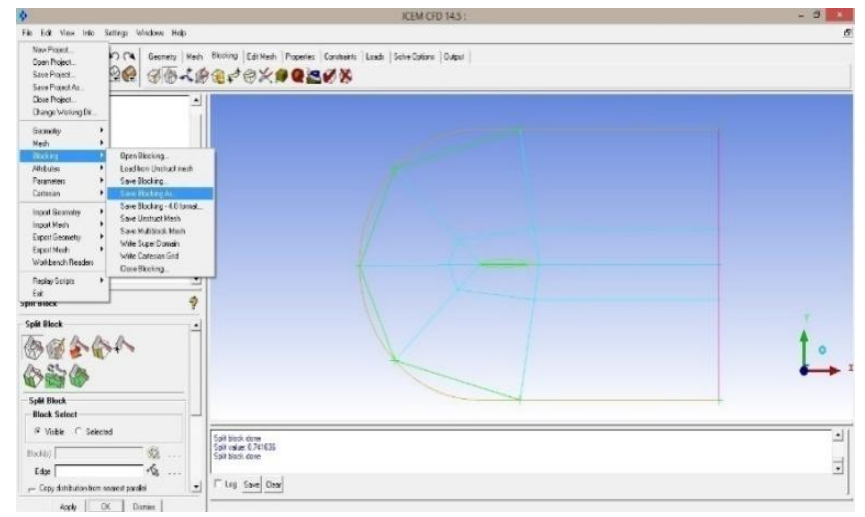

Fig 3.2(j) Saving Block

\subsection{Edge Parameters}

Edge parameters under the blocking menu is used for giving the number of nodes the edge is to be divided into and which bunching law it has to follow while dividing the edge.

\section{- $\quad$ Blocking $\rightarrow$ Pre-mesh params $\rightarrow$ Edge Params}

Select the edge and give the number of the nodes it has to be divided into as shown inbelow window fig 3.3(a). We can also select the type of bunching law it has to follow like uniform, geometri 1 , geometri 2 , etc... from the drop down menu. Simillarly give the edge parameters for all the edges and apply.

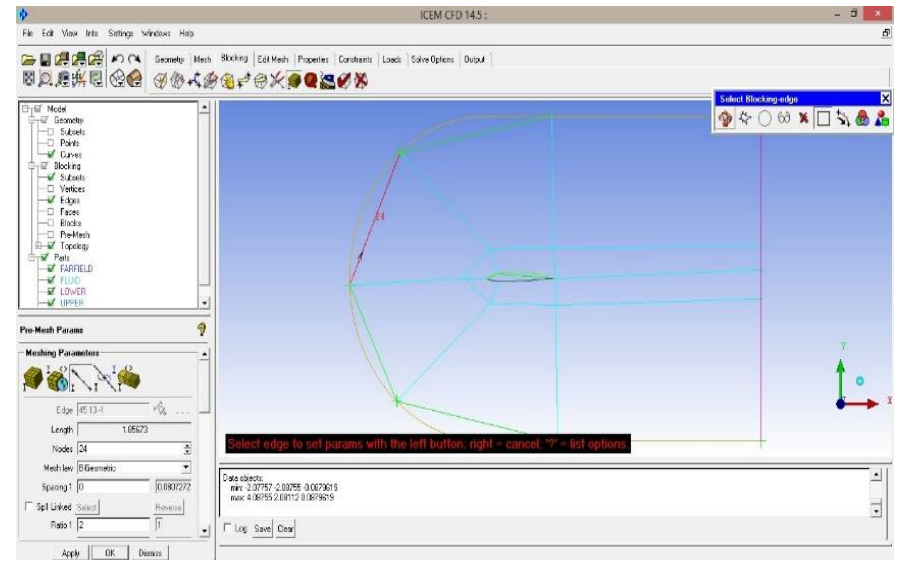

Fig 3.3(a) Giving Edge parameters

After giving the edge parameters, in the flow tree, right click on the pre-mesh under blocking and click on the recompute and give apply. Now we can see the mesh generated on the screen as shown in below figure 3.3(b)

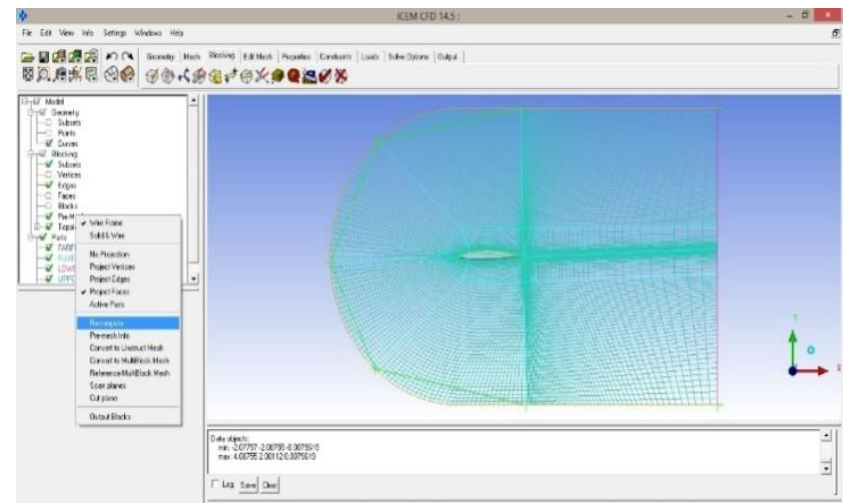

Fig 3.3(b) Mesh generated on Airfoil

Now the mesh is generated on the geometry. To go ahead, the quality of the mesh is to be checked. The ICEM CFD offers a variety of quality parameters to check mesh quality, in the drop down menu of quality criterion. Generally we check Angle and Determinant. The quality options can be seen by going through following command line.

\section{- $\quad$ Blocking $\rightarrow$ Pre-mesh Quality $\rightarrow$ Quality}

The Drop down menu of the quality criterion under the premesh quality is shown in below window fig 3.3(c). The quality criterion Angle of the mesh is checked and found to be good. The Angle criterion mesh check result is shown in below fig 3.3(d).

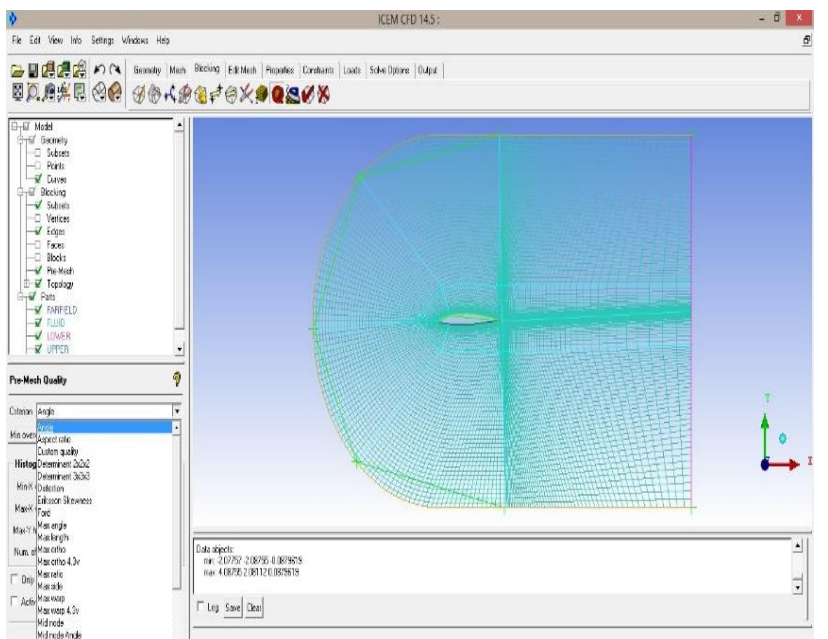

Fig 3.3(c) Premesh Quality checking

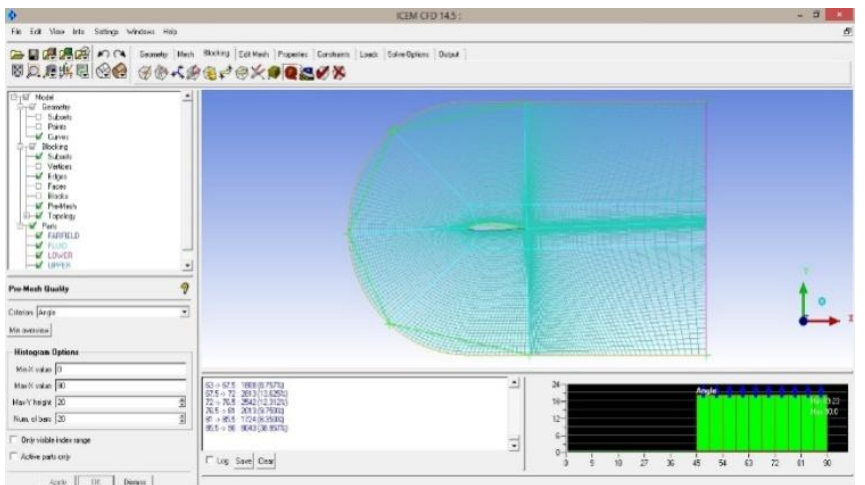

Fig 3.3(d) Premesh Quality criterion ANGLE 
The quality criterion - Determinant of the mesh generated is checked and found to be good. The Determinant criterion check result is shown in below fig 3.3(e)

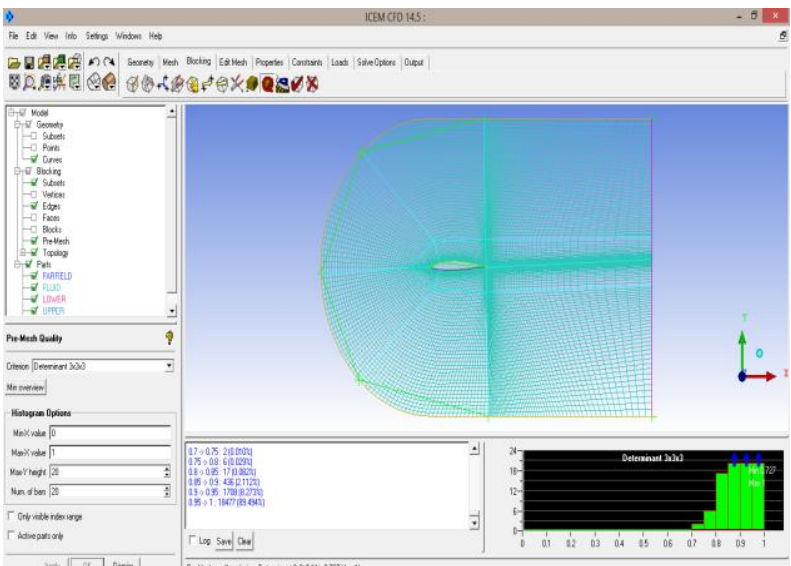

Fig 3.3(e) Premesh Quality criterion determinant

After checking the quality of the mesh generated and if it is found to be good, the mesh has to be exported to solver. The Fluent software is used for solving. The fluent reads the mesh in unstructured format only. So the genearated structured mesh has to be converted into unstructured format by following the command line below as shown in fig 3.3(f)

- $\quad$ Blocking $\rightarrow$ Pre-mesh $\rightarrow$ Right click $\rightarrow$ Convert to Unstructured mesh

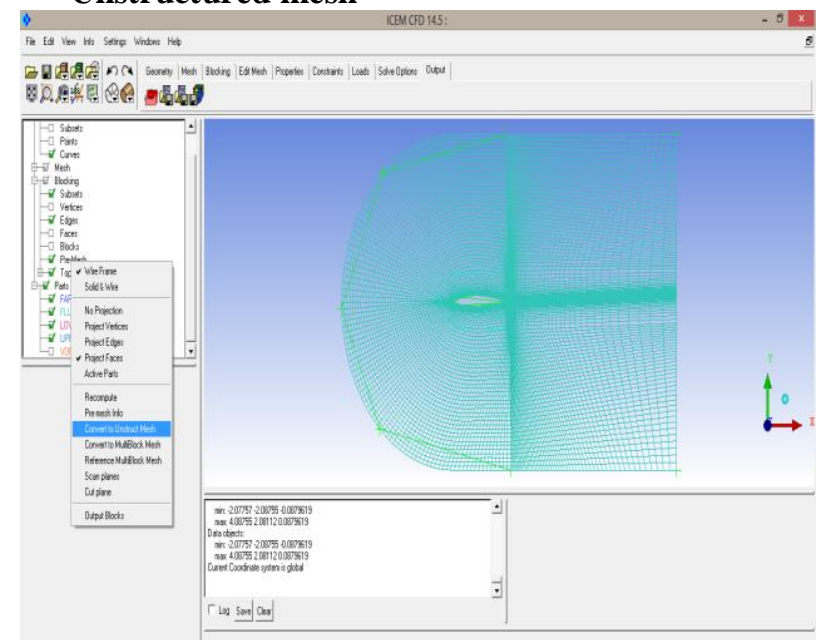

Fig 3.3(f) Converting to Unstructured mesh

The boundary conditions of each part has to be given before exporting. The solver type under the output should be selected first so that the setteing required for that solver are given in background. The below fig $3.3(\mathrm{~g})$ show the boundary condition window.

The farfield should be given boundary condition of Pressure farfield, fluid should be given as fluid and rest of the parts are given as wall. The command line is given below.
- $\quad$ Output $\rightarrow$ Family Boundary Conditions

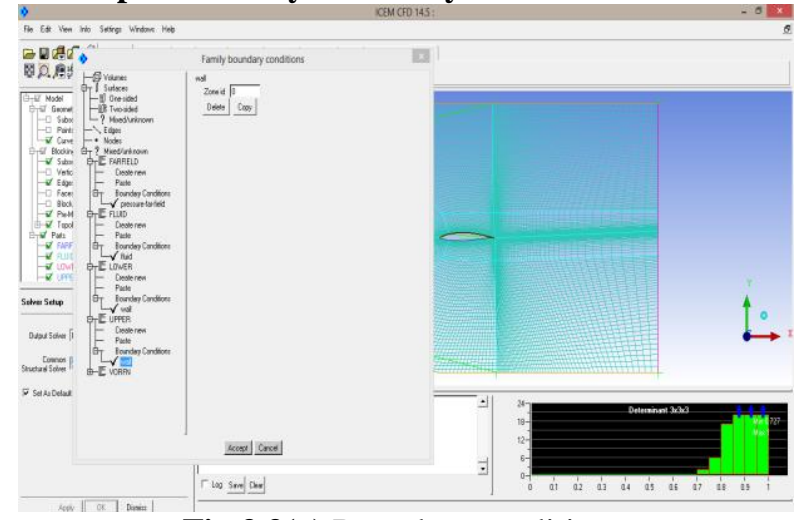

Fig 3.3(g) Boundary conditions

Now the mesh setting like 2D or 3D, Scaling, name and saving directory should be given in output window as shown in below fig 3.3(h)

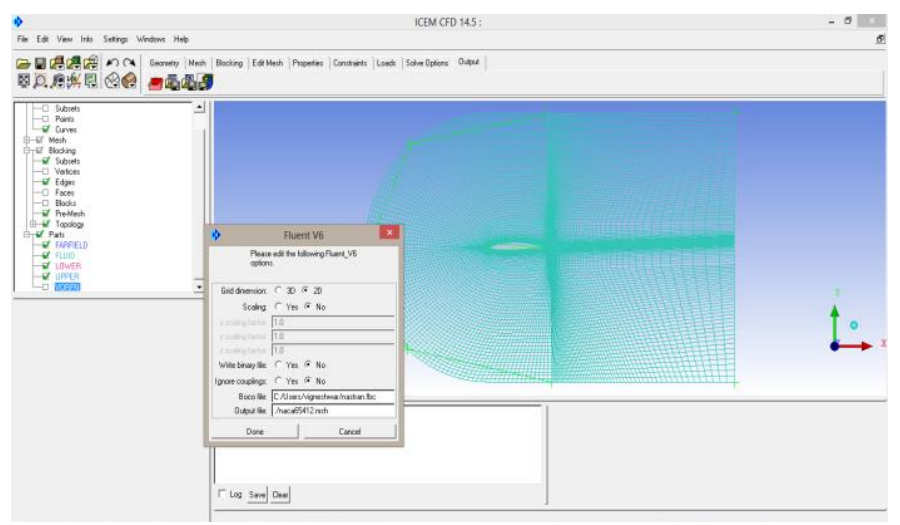

Fig 3.3(h) Exporting into .msh format

Now the mesh is ready to get imported into the solver.

\subsection{Fluent Solver Steps}

\section{- $\quad$ File $\rightarrow$ Import $\rightarrow$ Mesh $\rightarrow$ General}

The mesh can be imported using the above command. Now we can see the mesh in the window. The mesh should be scaled into meters and then mesh check has to be performed and report quality should be given. The minimum orthogonality quality is shown in the range of 0 to 1 . If the orthogonality quality is close to 0 the mesh quality is low. It is shown in blow fig 3.4(a)

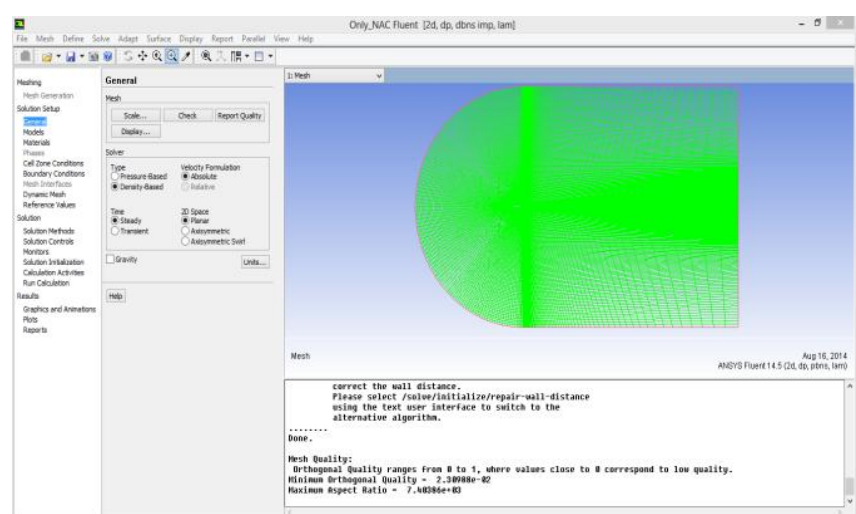

Fig 3.4(a) Mesh imported into fluent 
The solver settings should be given now. The Density based solver is choosed and the other parameters are given as shown in below fig 3.4(b)

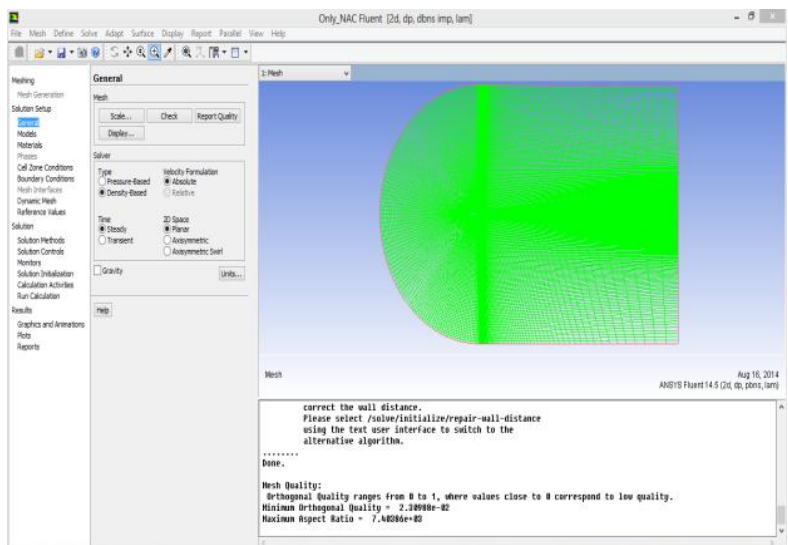

Fig 3.4(b) Mesh checked.

- $\quad$ Model $\rightarrow$ Energy Equation $\rightarrow$ On

- $\quad$ Model $\rightarrow$ Viscous $\rightarrow \mathrm{K \omega} \omega$-SST $\rightarrow$ Apply

- $\quad$ Material $\rightarrow$ Fluid $\rightarrow$ Air $\rightarrow$ Ideal gas $\rightarrow$ Apply

- Cell Zone Conditions $\rightarrow$ Operating Pressure $\rightarrow 0$ $\mathrm{Pa} \rightarrow$ Apply

Boundary Conditions $\rightarrow$ Check Farfield Condition $\rightarrow$ Edit The run conditions menu is seen by upper command line and run conditions are given as shown in below fig 3.4(c). The gauge pressure, mach, temperature and angle of attack values should be given. The gauge pressure is calculated with respect to the indian standard atmosphere.

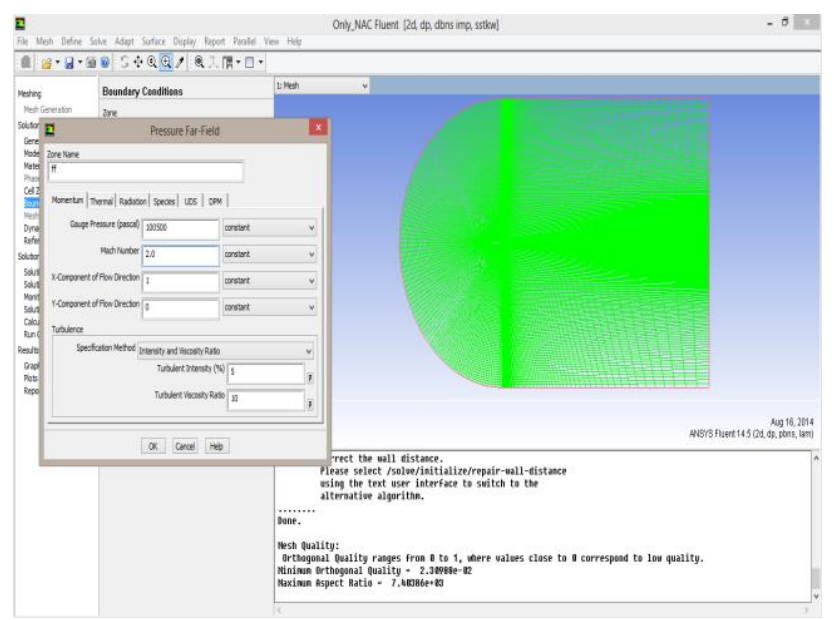

Fig 3.4(c) Simulation conditions

\section{- $\quad$ Reference Values $\rightarrow$ Compute from $\rightarrow$ Farfield(ff)}

Give the values of aera of the geometry and the reference length. The remaining values are calculated automatically from previous data. The window is shown in below fig $3.4(d)$

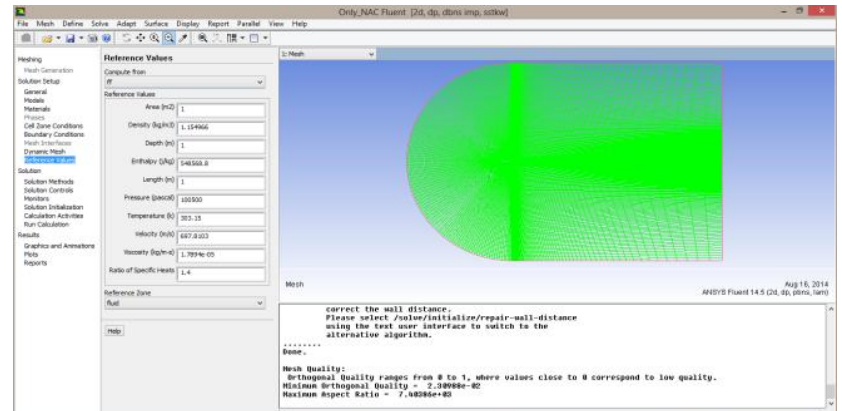

Fig 3.4(d) Reference values

- $\quad$ Solution Method $\rightarrow$ Formulation $\rightarrow$ Implicit

- $\quad$ Solution Method $\rightarrow$ Flux type $\rightarrow$ Roe-FDS

- $\quad$ Solution Method $\rightarrow$ Gradient $\rightarrow$ Green-Gauss Cell Based

The solution method window is shown in below fig 3.4(e). The above command options are selected from the drop down menus as shown in figure. The flow order is to be choosed according to the requirement of the simulation.

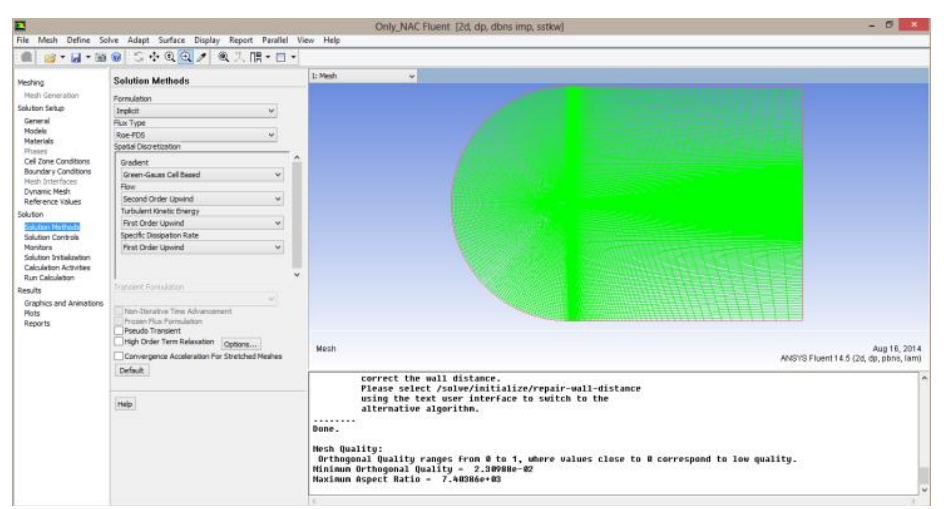

Fig 3.4(e) Solution methods

- Solution Controls $\rightarrow$ Courant Number $\rightarrow$ 2.5(default)

The courant number will have a high impact on the convergence of the simulation. The courant number should be given considering the mach number and the mesh quality for the simulation. The courant number should be reduced with increase in the mach number value. The courant number window is shown in below fig 3.4(f)

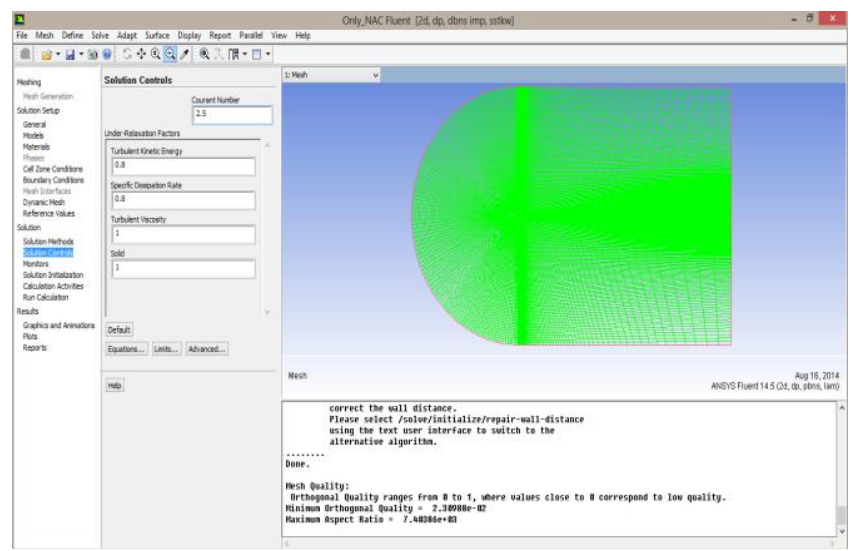

Fig 3.4(f) Solution controls 
- $\quad$ Monitors $\rightarrow$ Residuals $\rightarrow$ Print, Plot, Write

- Monitors $\rightarrow \mathrm{C}_{\mathrm{l}} \rightarrow$ Upper, Lower $\rightarrow$ Print, Plot, Write

- Monitors $\rightarrow \mathbf{C}_{\mathbf{d}} \rightarrow$ Upper, Lower $\rightarrow$ Print, Plot, Write

- Monitors $\rightarrow \mathbf{C}_{\mathbf{m}} \rightarrow$ Upper, Lower $\rightarrow$ Print, Plot, Write

The monitors are important to see the convergence of the simulation. The monitors can be seen as plots with respect to iterations and they can also be written to a file and saved.

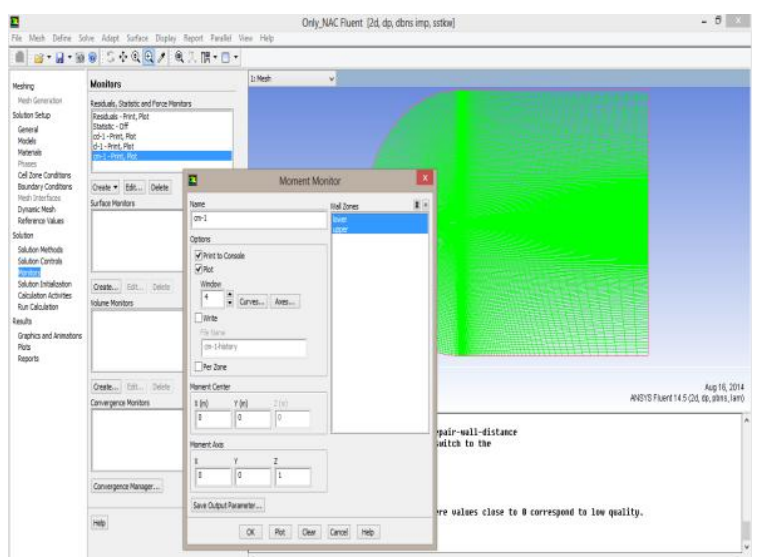

Fig 3.4(g) Simulation monitors

- $\quad$ Solution Initialization $\rightarrow$ Standard Intialization $\rightarrow$ Compute from $\rightarrow$ farfield $\rightarrow$ Initialize

For any simulation the initialization should be done after giving all the setting and method of solving before calculating the solution.

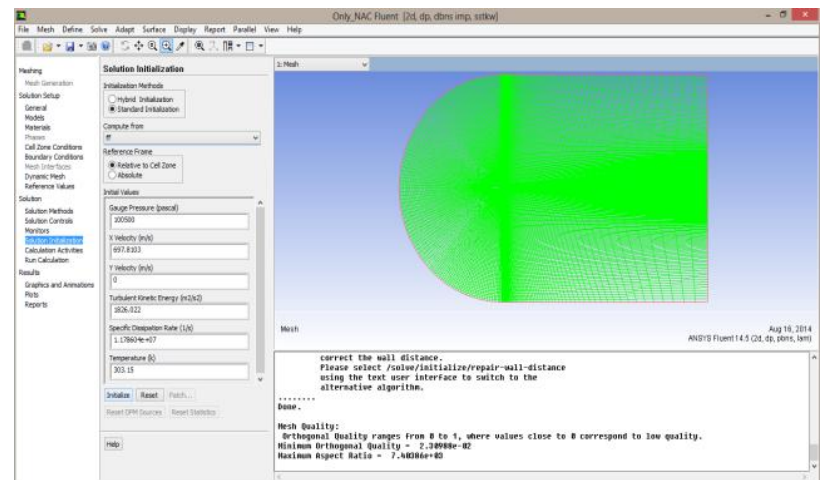

Fig 3.4(h) Simulation intialization

\section{- $\quad$ Run Calculations $\rightarrow$ Iterations $\rightarrow \mathbf{1 0 0 0} \rightarrow$ Run}

We can automatically save the simulation results by using the calculation activities in the flow tree. Otherwise we can save them manually using save case and that under the file menu. The case file will have the geometry details and the dat file will have all the simulation data. The result of the simulation can be seen through report and force report. The axial, normal forces and coefficients are obtained from the force report files which in return help to calculate the Lift and Drag.
Similarly the grid generation and simulations process is implemented for the Sharp spike and hemispherical spike geometries. The grids generated on both the geometries are shown in below fig 3.4(i)

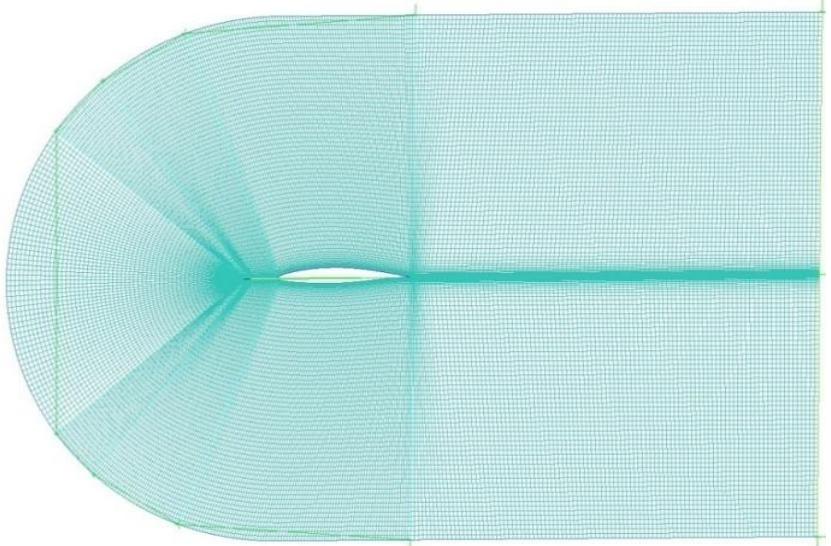

Fig 4.4(i) Sharp edged spike integrated to airfoil

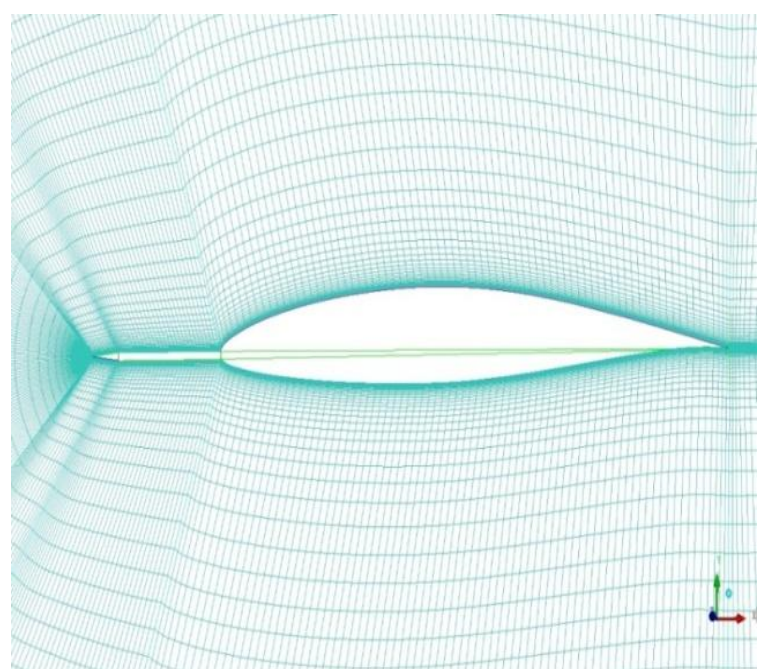

Fig 3.4(j) Sharp edged spike integrated to airfoil

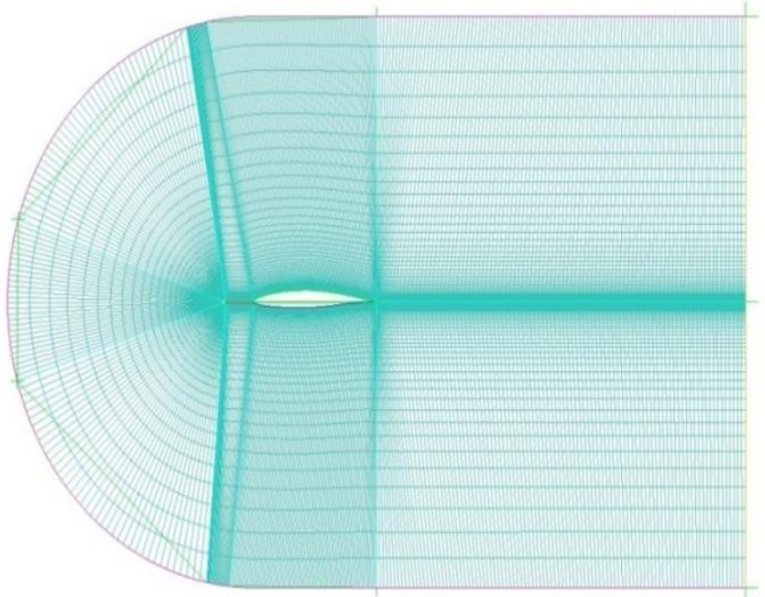

Fig 3.4(k) Hemi Spherical edged spike integrated to airfoil Zoomed view 


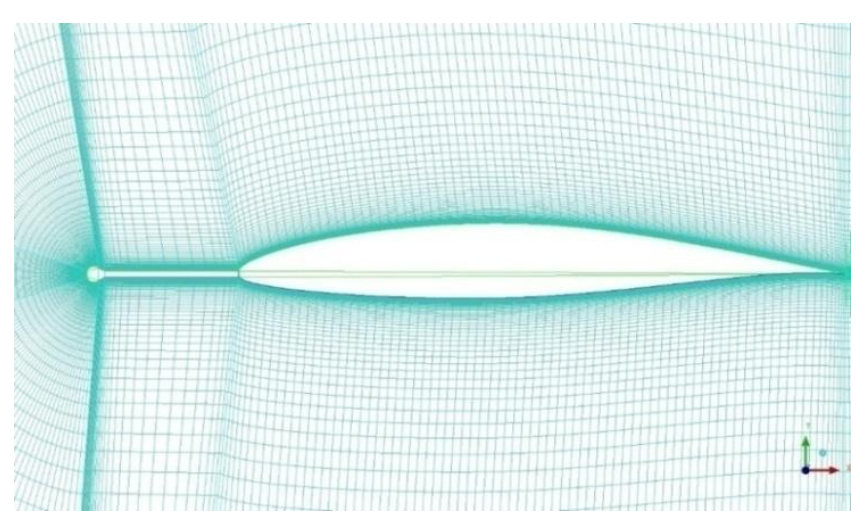

Fig 3.4(l) Hemi Spherical edged spike integrated to airfoil Zoomed vie

\section{RESULTS}

\subsection{Contours}

These are the Contours resulted after completion of simulation process in ANSYS Fluent.

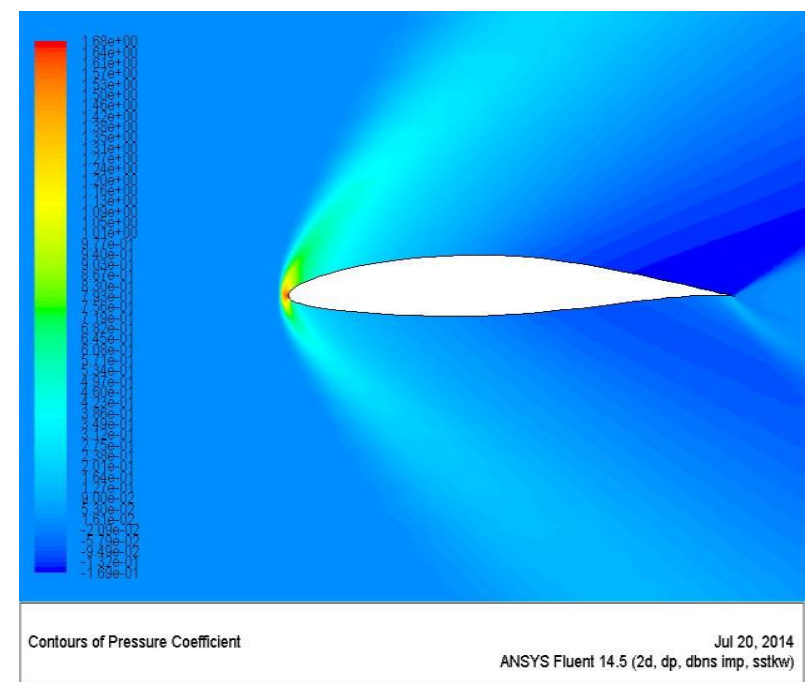

Fig 4.1(a) Pressure Coefficient contour over NACA 651412

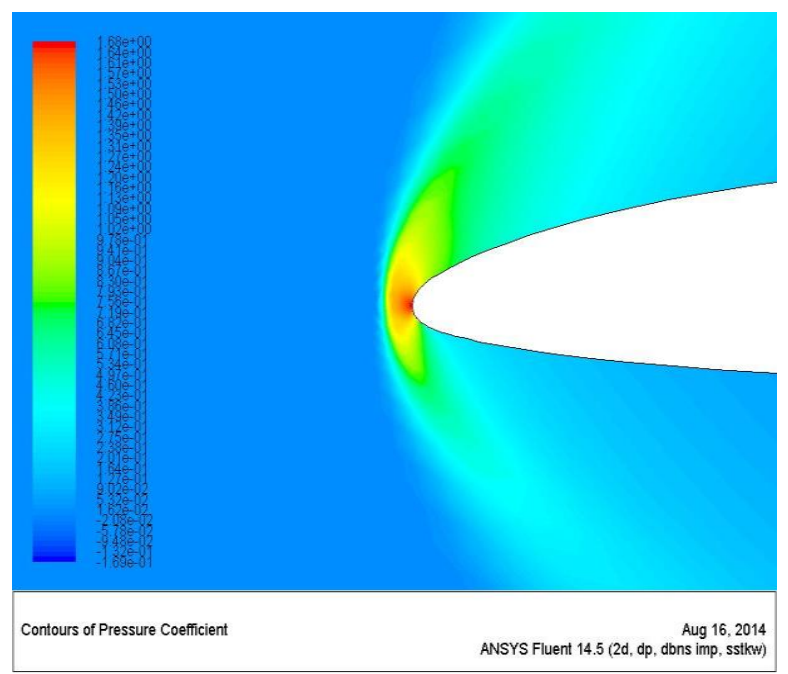

Fig 4.1(b) Pressure Coefficient contour over NACA 651412 zoomed view

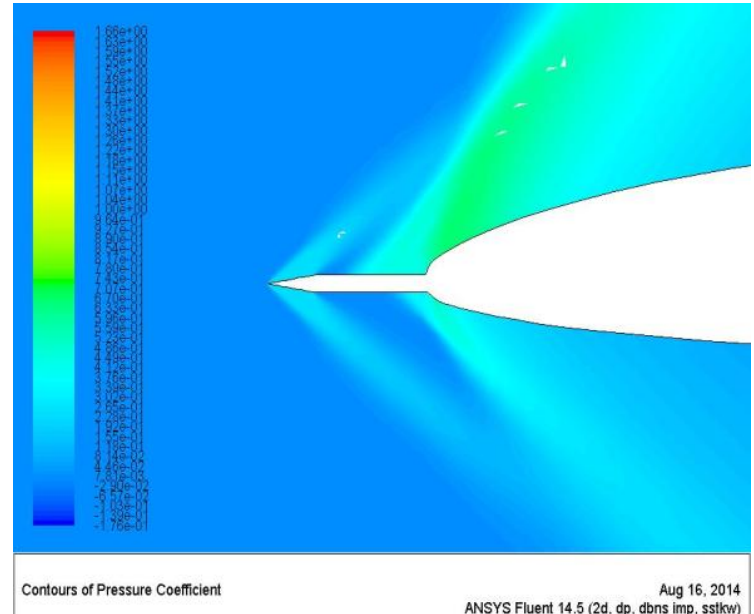

Fig 4.1(c) Pressure Coefficient contour over sharp spike integrated airfoil

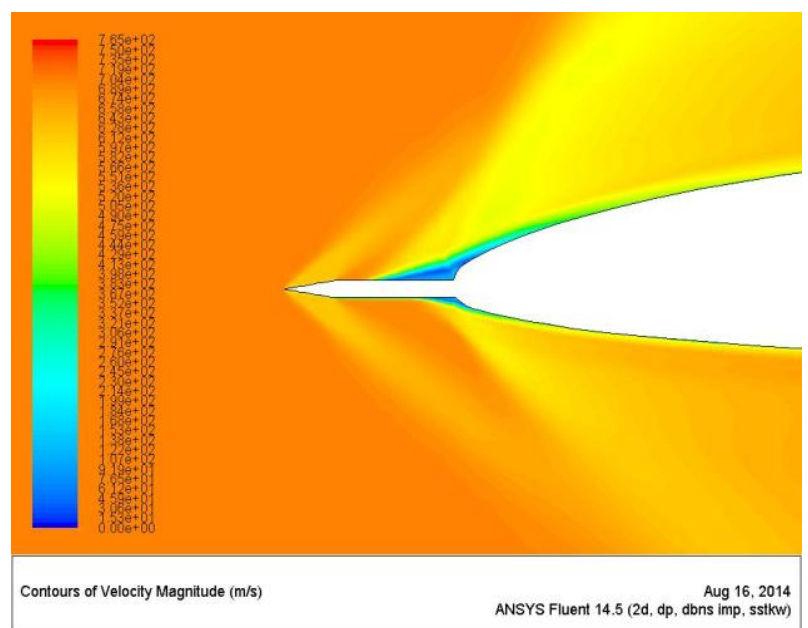

Fig 4.1(d): Velocity Magnitude Contour over sharp spike integrated airfoil

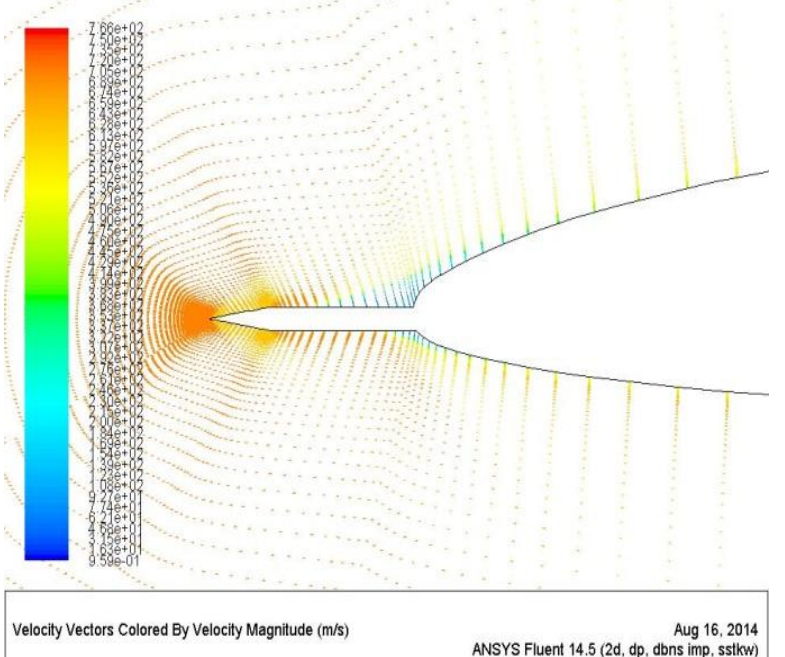

Fig 4.1(e): Velocity Vector of Velocity Magnitudeover sharp spike integrated airfoil 


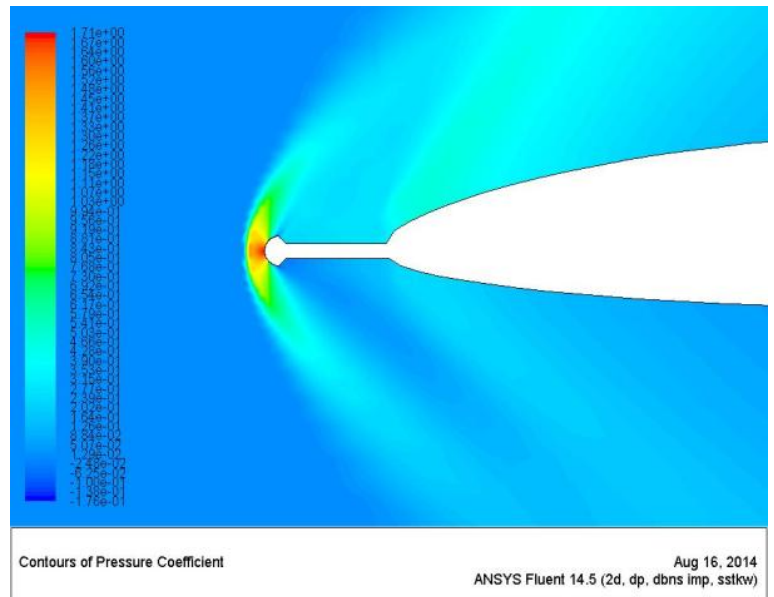

Fig 4.1(f): Pressure Coefficient contour over hemispherical spike integrated airfoil

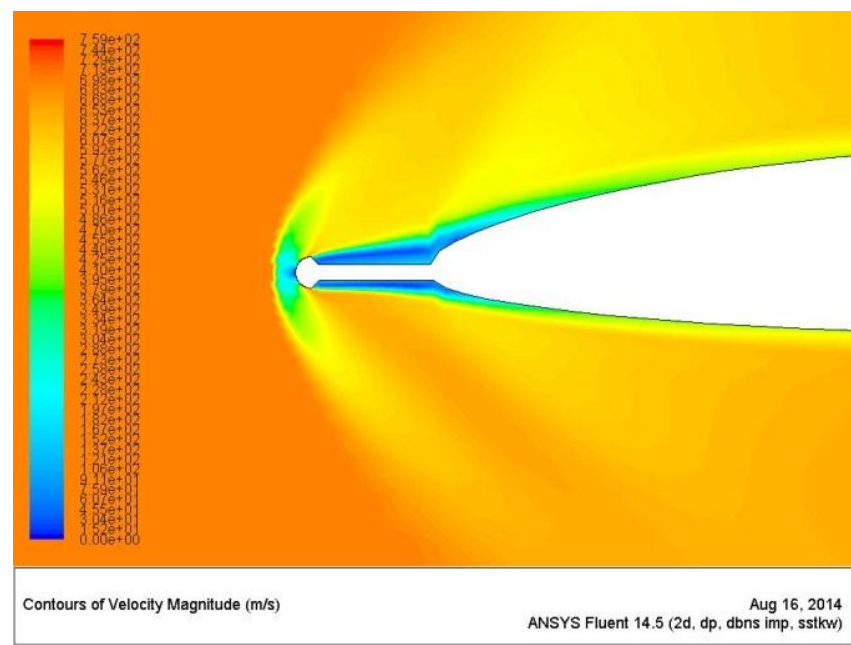

Fig 4.1(g): Velocity Magnitude Contour over hemispherical spike integrated airfoil

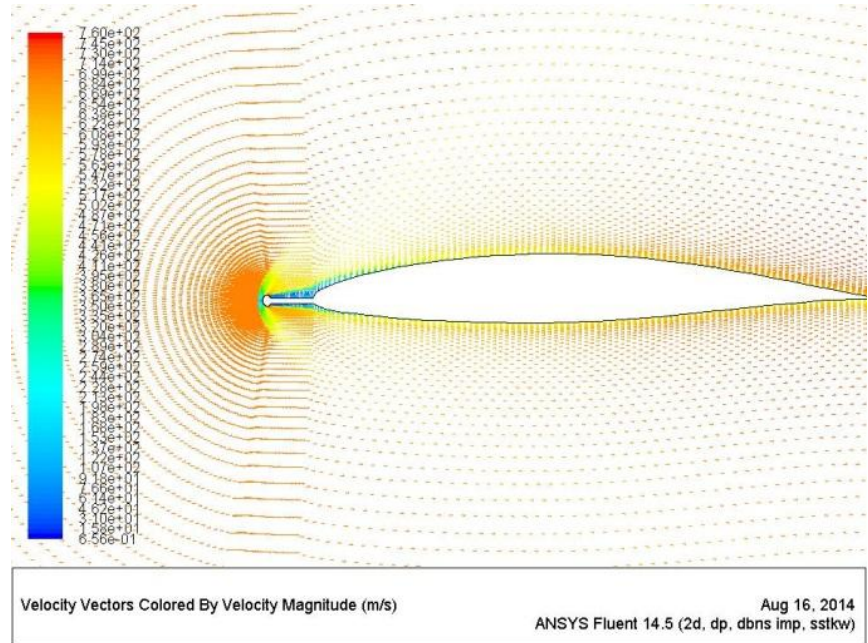

Fig 4.1(h): Velocity Vector of Velocity Magnitudeover hemispherical spike integrated airfoil

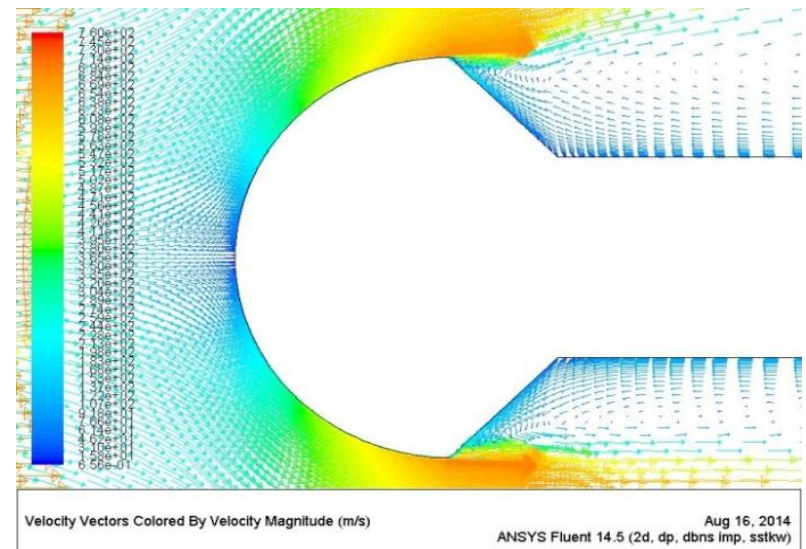

Fig 4.1(i): Velocity Vector of Velocity Magnitude over hemispherical spike integrated airfoil zoomed view

\section{CONVERGENCE}

The simulations were run until the converged solution is obtained. The convergence plot shows the simulation convergence. The convergence plot is obtained by plotting the iterations with respect to coefficient. The convergence plots of the NACA $65_{1}-412$ simulation are shown in below fig 5.1 and fig 5.2

\section{LIFT CONVERGENCE}

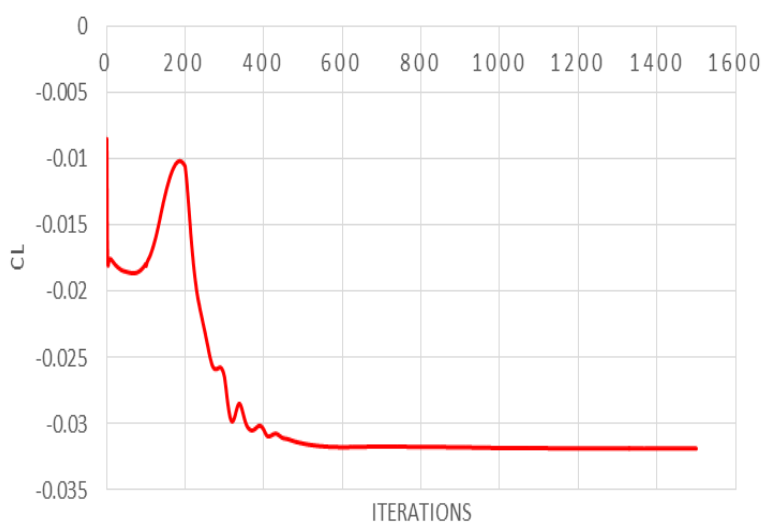

Fig 5.1 Lift Convergence

DRAG CONVERGENCE

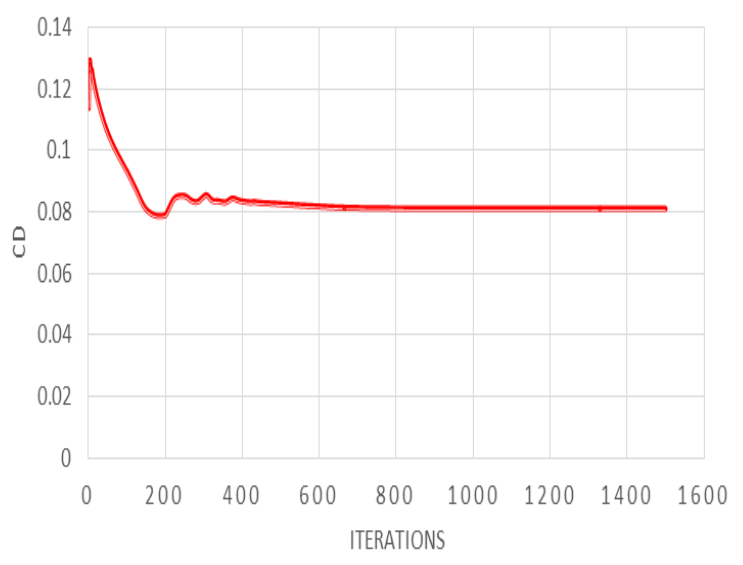

Fig 5.2 Drag Convergence 


\section{Calculations}

The Axial and Normal Forces and coefficients are obtained from the simulation results through force report files. These values are now used to calculate the lift and drag generated due to the supersonic flow over the airfoils. The comparison is made between the normal airfoil and two airfoils with spikes to see the change in the lift and drag forces, i.e. the aerodynamic loads. The force value of the sharp spike is also compared with the hemispherical one to find the effect of shape of spike on the aerodynamic loads. The formula used for the calculations and the calculated values are shown below

$\operatorname{Drag}(\mathrm{D})=N * \sin (\alpha)+A * \cos ((\alpha)$

Where, $\mathrm{N}=$ Normal Force,

$\mathrm{A}=$ Axial Force,

$\alpha=$ Angle of attack

Table: Calculation

\begin{tabular}{|l|l|l|l|}
\hline & $\begin{array}{l}\text { Normal } \\
\text { Airfoil }\end{array}$ & $\begin{array}{l}\text { Sharp } \\
\text { Spike }\end{array}$ & $\begin{array}{l}\text { Hemispherical } \\
\text { spike }\end{array}$ \\
\hline Axial Force & 22755.33 & 16761.71 & 19744.23 \\
\hline $\begin{array}{l}\text { Normal } \\
\text { Force }\end{array}$ & -8961.96 & 11077.2 & 9497.34 \\
\hline $\begin{array}{l}\text { Drag (at } \alpha= \\
\left.0^{0}\right)\end{array}$ & 22755.33 & 16761.71 & 19744.23 \\
\hline $\begin{array}{l}\text { Coefficient } \\
\text { of Drag }\end{array}$ & 0.080994 & 0.059608 & 0.070214 \\
\hline
\end{tabular}

\section{DISCUSSION}

Experiments have been made to visualize the overall flow field over NACA 651-412 aerofoil with and without spike. The simulation velocity contours pictures shown indicate a strong detached bow shock wave in front of NACA 6 series aerofoil as expected. With adoption of sharp spike, a conical shock is observed and with hemi spherical spike, a bow shock is observed. The closer look of velocity vector on all the cases studied, the flow is reversed. The measured drag $\left(C_{D}\right)$ for NACA 6 series aerofoil with adoption of sharp spike is much decreased than hemi spherical spike adopted case. This indicates that, use of any of these spikes lead to substantial reduction in drag.

The pressure contours for all the cases are shown in fig. 3 along with typical values of pressure. Pressure is observed to be maximum at Leading edge of NACA 6 series aerofoil. For sharp spike case, low pressure effect is observed on spike as well as on NACA 6 series aerofoil. But in hemi spherical case, pressure values too high at head of hemi spherical spike as well as at Leading edge of aerofoil.

The main reduction in drag is due to reduction in pressure drag on the aerofoil with change in spike. This indicates that the further reduction in drag could be achieved by suitably modifying the sharp spike mainly and as well as hemi spherical tip of spike.

\section{CONCLUSION AND FUTURE SCOPE}

Computations have been made to obtain the flow field on a NACA 651-412 aerofoil at a supersonic Mach number of 2, in the presence of a sharp spike and hemi spherical head spike. It was observed that in general the drag reduces. Computations results indicate more details of flow field and effect of spike shape and its length with and without spike mode.

Estimation of drag for different components indicate that major reduction is from the NACA 6 series aerofoil which get modified due to spike tip and hence shape and size of spike is of prime importance for reduction of drag.

\section{FUTURE SCOPE OF WORK}

- $\quad$ To implement the spike to 3D wing geometry and study the effect of spike on the complete wing.

- To implement the spike to supersonic aircraft wings and compare its effect.

- $\quad$ To increase the performance of aircraft by making it capable to fly in both subsonic and supersonic flight speeds

- $\quad$ To reduce the fuel usage in supersonic aircraft

\section{REFERENCES}

[1]. P.k.kundu, I.M.cohen. Fluid Mechanics 3/e. Academic press, Indian Reprint 2005.

[2]. Kalimuthu, r. and Rathakrishnan, E., "Aerospike for drag reduction in hypersonic flow", AIAA-2008-4707.

[3]. Kalimuthu,R., Mehta, R.C. and Rathakrishnan, E., "Drag Reduction for Spike Attached to Blunt-nosed Body at Mach 6", Engineering Notes, Journal of Spacecraft and Rockets, Vol.47, No.1, jan-Feb,2010,pp.219-222.

[4]. D'Humieres, G. and Stollery, J.1., "Drag Reduction on a Spiked Body at Hypersonic Speed", The Aeronautical Journal, Paper No.3482, Vol.114, No.1152, February, 2010, pp.113-119.

[5]. Ahmed, M.Y.M. and Qin, N., "Recent Advances in the Aerothermodynamics of Spiked Hypersonic vehicles", Progress in Aerospace Sciences, 47, 2011, pp.425-449.

[6]. Van Driest, E.R.: "The Problem of Aerodynamic Heating", Aeronautical Engineering Review, Oct. 1956, pp. 26-41.

[7]. Anderson, John D., Jr., Fundamentals of Aerodynamics, 3rd edi., McGraw-Hill Book Company, New York, 1984. 